\title{
Improvement of the Handover and Quality of Service on Software Defined Wireless Networks
}

\author{
Fatima Laassiri ${ }^{1, \mathrm{a}}$, Mohamed Moughit ${ }^{2, \mathrm{~b}}$ \\ ${ }^{\mathrm{a}, \mathrm{b}}$ IR2M Laboratory, FST, Univ Hassan UH1- Settat, \\ Morocco \\ ${ }^{\mathrm{b}}$ EEA\&TI Laboratory, FST, Univ Hassan, Mohammedia, \\ Morocco, ${ }^{\mathrm{b}}$ National Schools of Applied Sciences \\ Khouribga, Univ Hassan 1, UH1- Settat, Morocco
}

\author{
Noureddine Idboufker ${ }^{3}$ \\ National School of Applied Sciences, \\ Univ Cadi Ayyad Marrakech, \\ Morocco
}

\begin{abstract}
The Wireless Fidelity (WiFi) is the business name given to the 802.11b and 802.11g IEEE standard by the WiFi Alliance, formerly known as Weca industry with more than 200 member companies dedicated to supporting the growth of wireless LANs. This standard is currently one of the most used standards in the world. The theoretical data rates of $802.11 \mathrm{~b}$ are $11 \mathrm{Mb} / \mathrm{s}$ and $54 \mathrm{Mb} / \mathrm{s}$ for 802.11g. This article presents Handover's improvement performance and quality of service (QoS) parameters and they are: end-to-end delay, latency, jitter, lost packets, and Mean Opinion Score (MoS), under networks Wi-Fi with the help of the OMNeT $4.6++$, by implementation of a new algorithm at the level of the SDN controller that allows handover management without breaking the connection by respecting the priority per class of traffic. The realization of this work is based on the intra-Wi-Fi mobility, that it is adopted by a macro mobility of level 3 and it is MIPv6 as well as it exploited the protocol of Voice over IP that it is SIP, and the implementation of SDN rules on the OpenFlow protocol.
\end{abstract}

Keywords-SDN; Wi-Fi; QoS; OpenFlow protocol; handover; SDN controller; OpenFlow switch

\section{INTRODUCTION}

Software Defined Network (SDN) [1] is a new network paradigm; it is used to reduce the complexity of network technology. The following work aims to expose a simulation implemented under OMNeT 4.6++, to assess the performance of Handover and the QoS with two architectures one Wi-Fi [2] without SDN and the second offers the improvement of the implementation the SDN via the OpenFlow protocol [3].

The communication between the two topologies is carried out with the SIP protocol [4], with high quality intra-Wi-Fi mobility that is MIPv6 [5].

Today, we are seeing the evolution of the Internet in a number of users. Among the factors of this evolution is the success of 802.11. The IEEE 802.11 networks are becoming increasingly popular as they allow users to connect to the Internet at an affordable price with relatively large bandwidth and the ability to roam without being disconnected. In addition, nowadays IEEE 802.11 wireless network cards are deployed in the majority of technologies such as PDAs and laptops relatively important and also the ability to move without being disconnected. In addition, nowadays IEEE
802.11 wireless network cards are deployed in the majority of technologies such as PDAs and laptops.

In parallel, multimedia communication techniques have also evolved with the new compression and coding algorithms. Thus, many multimedia applications become accessible from wireless networks. But they still present obstacles to deployment. The major problems of these networks are the lost rate and the delay variation knowing that multimedia applications are very demanding. An obvious solution to optimize bandwidth utilization and improve video quality is to transmit multi-point video to a set of users.

But the use of standard Multipoint has three main problems. The first is the impossibility of adapting the collision windows according to the state of the network. The second is the impossibility of adapting the physical rate according to the state of the transmission medium, sod the packets are transmitted at a fixed physical rate. The third is the impossibility of retransmitting lost packets at the MAC layer.

A new approach has recently been proposed to remedy its problems. It consists of the election of a receiver called leader to ensure the acknowledgment of received packets. Thus, the transmitter can adapt to the physical rate and retransmit the lost packets.

\section{PRoblem AND SOlutions}

Wi-Fi refers to certain types of Wireless Local Area Networks (WLANs) [6] and uses specifications that fall under the 802.11 standard [7]. It is widely used by companies, because of the growing demand, users have ubiquitous access to wireless services, what it led to the deployment of forced use of this wireless access technology such as Wi-Fi, it offers a level of quality within reach, but the problem is that the number of devices is increasing which reduces the performance of travel time Handover and QoS in terms of end-to-end delay, latency, jitter, the number of lost packets, and $\mathrm{MoS}$, for all these reasons, this work proposes as a solution to implemented SDN technology for Wi-Fi to better optimize performance.

\section{STATE OF THE ART}

Before the appearance of SDNs, they are defined by the Open Networking Foundation (ONF) [8] as we know them today, several ideas and works have been proposed before, 
including network programming, separation of control and data designs. This segment is a brief overview of this work, that he can be considered as ancestors of SDN. The first idea of programming networks was developed in 1996 under the name of "Active Network" (AN) [9]. These networks infusion programs among the packet data. When a network node receives packets, it extracts and executes the programs from the data of the packet and therefore triggers standard activities of transmission, adjustment or concealment of the packet. With this approach, new network administration and routing mechanisms can be implemented without altering transmission equipment. Several studies have been conducted on NAs, especially on smart packets [10]. Since packet can carry malicious programs, and an elective called "Programmable Networks" (PN) [11] was proposed in 1999. The PNs inject programs inside the nodes of the network. These nodes run the programs only after a signalling and verification stage, to enhance security. ANs and PNs have sought to introduce programmability into networks through packets and programmable switches. These approaches did not reduce the complexity of the network infrastructure. In 1998, the Internet Engineering Task Force (IETF) working group could propose a General Protocol for Managing Switches (GSMP) [12], also another project called 4D was launched in 2005 [13] to separate the routing decisions and the protocols that govern the connections between network devices. Dispersal and discovery designs collect information from the network and send it to the decision plan, which has a global view of the network, to control the transmission of traffic flowing through the data model. The beginning of the SDN networks started with the Ethane project [14], launched in 2006 at the University of Stanford. In fact, that it defines a new engineering for business networks. Ethane goal was to have a centralized controller to manage the rules (Arrangements) and security in the network. Ethane uses two components: A controller to decide whether a packet should be forwarded, and an Ethane switch consisting of a table and a match string between the two. He was a source of inspiration for a networking operating system called Nox [15], and for a new idea called today Software Defined Network (SDN). Noting that Ethane's researchers are behind Nox and SDN [16].

\section{IEEE 802.11 STANDARDS}

IEEE $802.11 \mathrm{ac}$ is a wireless standard for the Wi-Fi family, standardized by IEEE; it allows a high-speed wireless connection to a local area network and uses only a frequency band between 5 and $6 \mathrm{GHz}$, with variations depending on the country. This frequency band is commonly named: "band of $5 \mathrm{GHz}$ " [17].

The aggregated channels allow, under ideal radio conditions, a theoretical throughput of up to $1.3 \mathrm{Gbit} / \mathrm{s}$ and the throughput of $910 \mathrm{Mbit} / \mathrm{s}$ (using four channels occupying an $80 \mathrm{MHz}$ sub-band), up to $7 \mathrm{Gbit} / \mathrm{s}$ overall throughput [18].

\section{OPENFLOW PROTOCOL}

The OpenFlow protocol is defined by the ONF, this nonprofit consortium dedicated to the development and standardization of SDN. It uses the TCP protocol via port 6633[19]. The communication uses a secure channel based on TLS.
OpenFlow protocol supports three types of messages [20]:

Controller to switch messages: These messages are sent only by the controller to the switches, they perform the switch configuration functions, they exchange information about the capabilities of the switch and they also manage the flow tables.

Symmetrical messages: These messages are sent back and forth signalling the connection problems of the switch controller.

Asynchronous messages: These messages are sent by the switch to the controller to announce changes in the network and switch status. [20]

\section{A. Layers of SDN}

Fig. 1 offers the general architecture of the SDN layers and the OpenFlow protocol.

Infrastructure layer [21]: For an OpenFlow protocol implementation function of a network element, the part of the equipment that it provides an API and an interface to the controller.

Control layer[22]: Responsible for making decisions about how packets should be transmitted by one or more network devices, and push those decisions to network devices for execution.

Northbound SDN Interfaces (NBI) [23]: NBI interfaces of SDN are interfaces between SDN applications and controllers, they typically provide abstract network views, and they allow direct expression of network behaviour and requirements.

Applications SDN [24]: This layer is a program; it communicates the necessary behaviours and resources with the SDN controller through application programming interfaces (APIs). In addition, applications can build an abstract view of the network by gathering information from the controller for decision-making purposes.

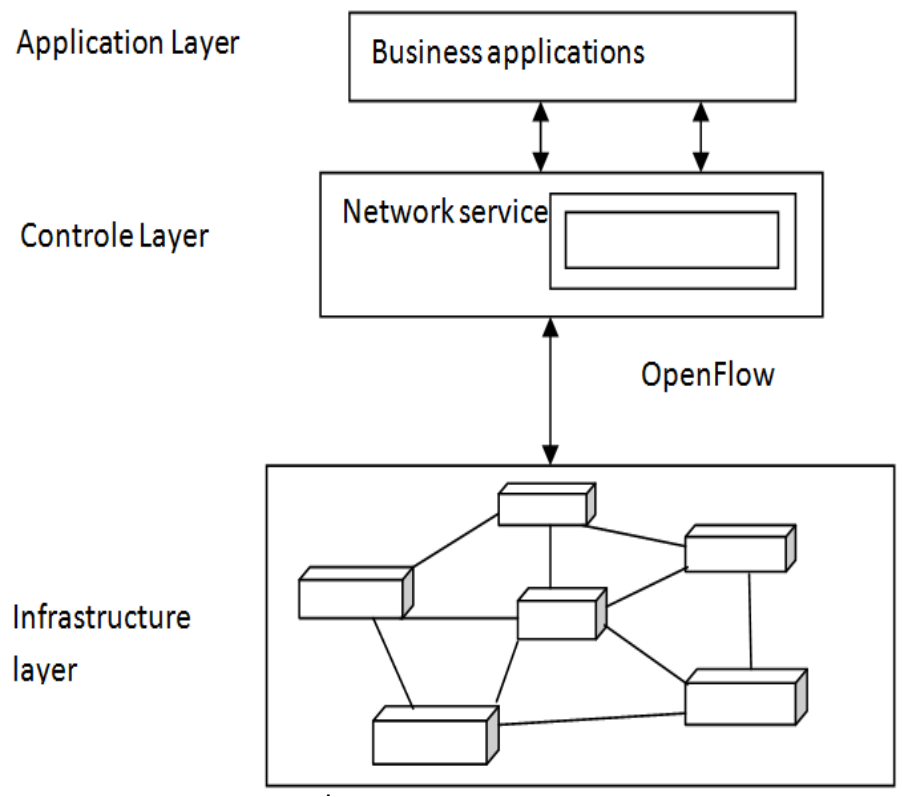

Fig. 1. SDN Network Architecture. 


\section{METHODS FOR IMPLEMENTING THE WI-FI WITH AND WITHOUT SDN UNDER OMNET 4.6++}

This section describes two simulation topologies implemented under OMNeT 4.6++, with the first presenting a Wi-Fi network without SDN (Fig. 2) and the same with the addition a new algorithm at the Controller SDN, that it is based on the OpenFlow protocol ( Fig. 3).

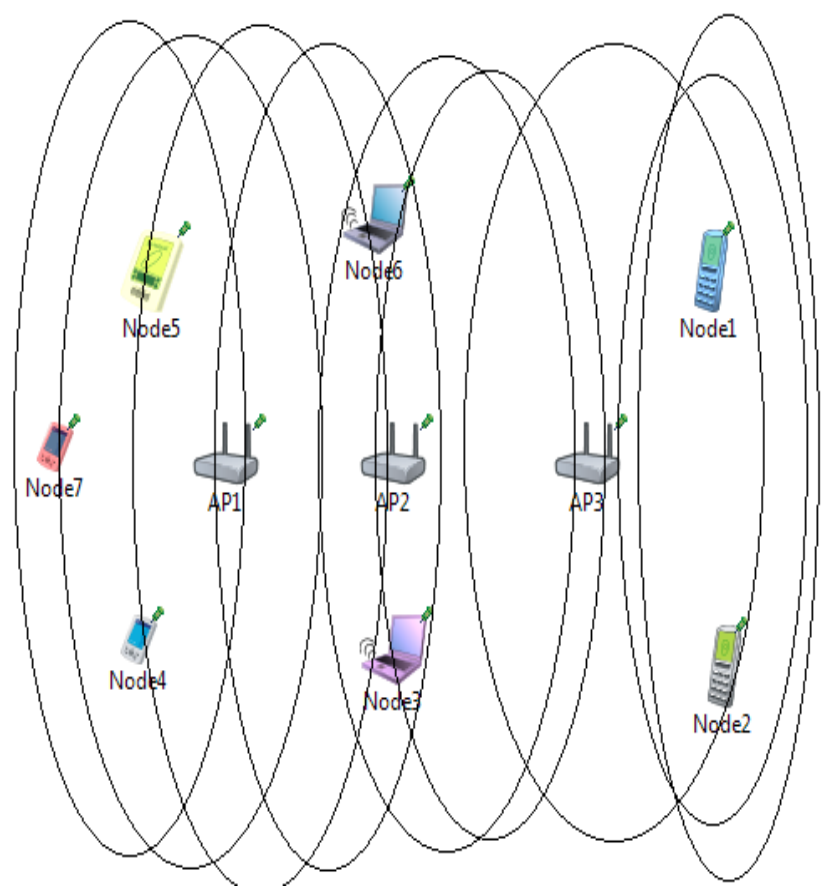

Fig. 2. Scenario 1: Wi-Fi Network Architecture without SDN.

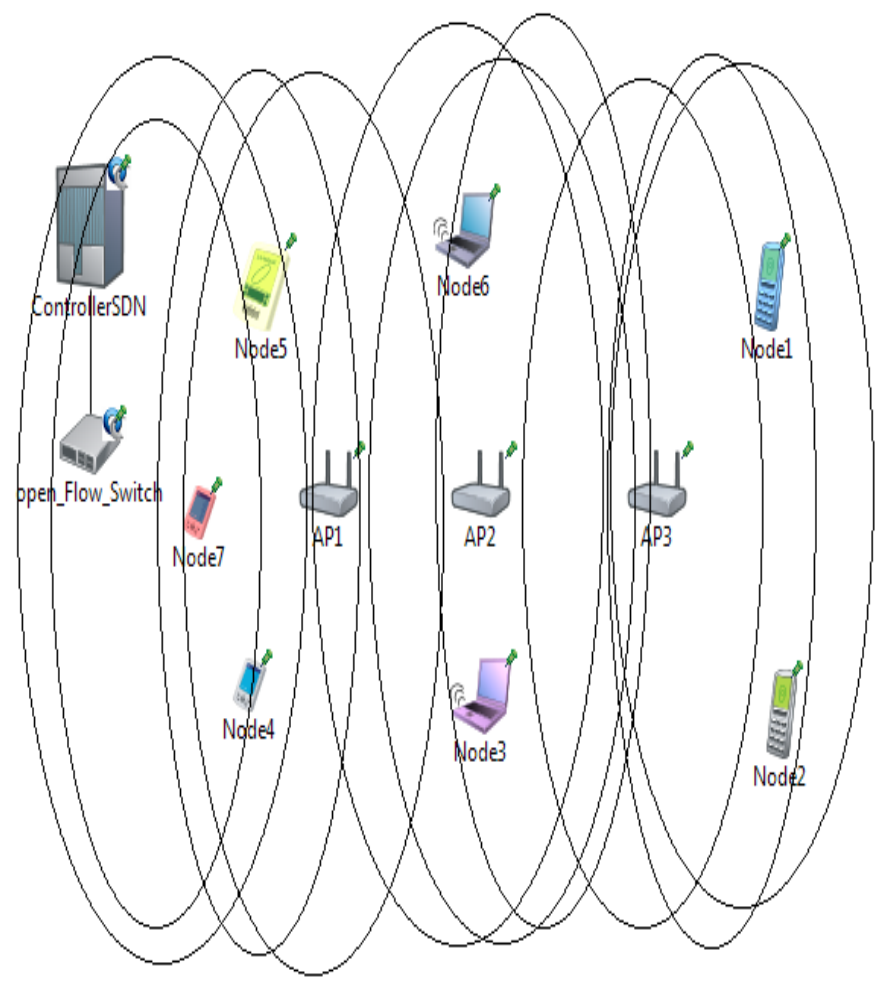

Fig. 3. Scenario 2: Wi-Fi Network Architecture with SDN.
For the realization of this work, the mobility used intra Wi-Fi, it occurs when a user device moves from one node to another, with a manipulation of a level 3 Macro Mobility, taking advantage of the MIPv6 protocol for mobile nodes to move randomly across the Internet, while continuing to receive their datagrams at a fixed address.

Each access point has at least two network interfaces: An 802.11 interface communicates with 802.11 clients and the second Ethernet-type interface connects to the main wired network. Packets received from the customer will be transmitted from one side to the other... Transmitting a package or not. It is determined by consulting the flow tables. That it consists of a set of rules, maintained by the controller via the interface OpenFlow. If the access point enabled by OpenFlow finds no corresponding rule in the flow table of a packet, it will ask the controller to process the packet.

The OpenFlow controller schedule coordinates the transmission between access points. The responsibility of scheduling algorithm is running in the controller and reducing the occurrence of conflicts and retransmission.

The OpenFlow interface is simply started, with the abstraction of a single table of rules it could match packets on a dozen header fields (MAC addresses, IP addresses, protocols, TCP / UDP Port numbers, etc.). In the last five years, the specification has become more and more folded, with many of the header fields and multiple stages of the rule tables, to allow the switches to expose more of their capabilities to the controller.

The OpenFlow specification allows future switches to support exile mechanisms to parse the corresponding packets and header fields, allowing controller applications to take advantage of these capabilities through a common open interface.[25]

\section{A. Components of the SDN Architecture with and without SDN}

The Wi-Fi architecture with and without SDN consists of the following:

Station (STA): Client device in an 802.11 (Wi-Fi) that it has chip and antenna such as a computer, a laptop or a smartphone. The term STA is sometimes used for the access point (AP), in which case an STA is a device communicating via the 802.11 protocol.

An access point $(A P)$ : Is a device of a wireless LAN, or WLAN, usually in an office or in a large building. It connects to a wired router, switch, or Ethernet cable hub and delivers a Wi-Fi signal to a dedicated zone.

SDN Controllers: To provide a layer of abstraction of the network and present it as a system. It allows to quickly implement a change on the network by translating a global demand (for example: Prioritize application X) in a sequence of operations on network devices (OpenFlow Additions States). Orders are given to the controller by an application via a so-called API "Northbound" or north. Controller software vendors publish the API documentation to allow applications to interface. The controller communicates with the equipment via one or more APIs called "Southbound" or south. 
OpenFlow is positioned as a south API acting directly on the data plane. Other APIs can act on the management plan or control. A controller can even speak directly in CLI with a device to activate a feature. [26]

OpenFlow switch: It is a physical switching device that contains a number of ports and queues, it is based on the OpenFlow protocol.

\section{RESULTS AND DISCUSSION OF SIMULATION IN QUALITY OF SERVICE CRITERIA (QOS)}

This section presents Handover and QoS performance evaluation such as end-to-end delay, latency, jitter, lost packet, and MoS. Under Wi-Fi without SDN and with the latter's increment to determine the impact of implementation the new algorithm in SDN controller.

A generic access point supports multiple wireless radios and multiple Ethernet ports. The Ethernet MAC type of the relay unit is wireless card type.

By default, the access point is stationary (Mobility Stationary), but it can also be configured by parameters.

\section{A. End-to-end Delay under Wi-Fi without and with SDN}

Fig. 4 shows the end-to-end delay results in the Wi-Fi without SDN scenario with a higher value of $(35 \mathrm{~ms})$ compared to the SDN-based scenario which has a reliable delay of $(22 \mathrm{~ms})$, which explains why adding the latter is beneficial.

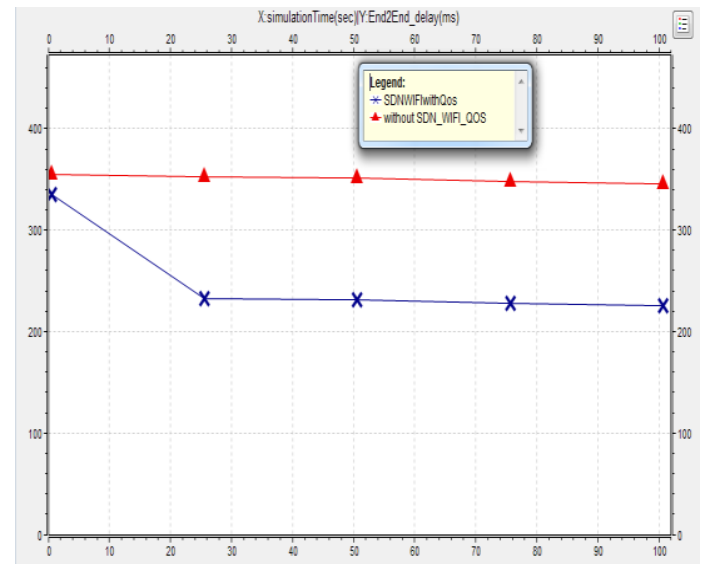

Fig. 4. End-to-end Delay in Wi-Fi SDN-Free and SDN-Based Scenarios.

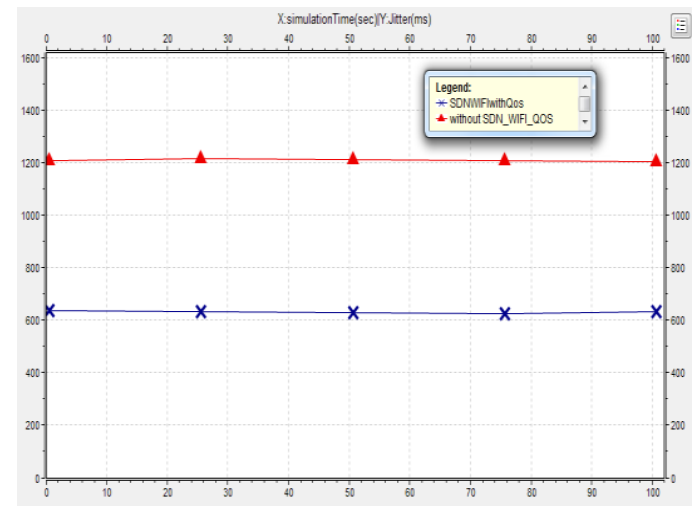

Fig. 5. Jitter in Wi-Fi SDN-Free and SDN-Based Scenarios.

\section{B. Jitter under Wi-Fi without and with SDN}

The jitter under Fig. 5 watch the Wi-Fi scenario based on SDN is about $60 \mathrm{~ms}$, it is half of Wi-Fi without SDN; it has the value of $12 \mathrm{~ms}$, which results that the addition of a SDN network for Wi-Fi is fruitful.

\section{Latency under Wi-Fi without and with SDN}

The results of Fig. 6 shows that the Wi-Fi network latency with SDN is lower $(55 \mathrm{~ms})$ than that of the Wi-Fi approach without SDN with a value of $125 \mathrm{~ms}$, which justifies that SDN adds a positive appreciation for $\mathrm{Wi}-\mathrm{Fi}$.

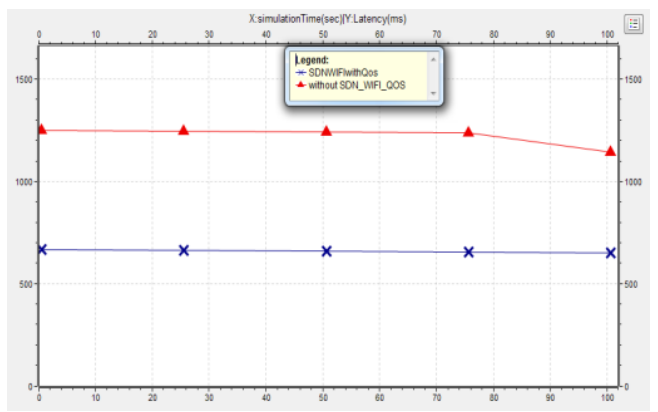

Fig. 6. Latency in Wi-Fi SDN-Free and SDN-Based Scenarios.

\section{Packets lost under Wi-Fi without and with SDN.}

The number of packets lost in the Wi-Fi approach without SDN is $75 \%$, it is higher compared to the Wi-Fi approach with SDN, which is about $62 \%$. This shows the impact of adding the SDN approach to the Wi-Fi network, as shown in Fig. 7.

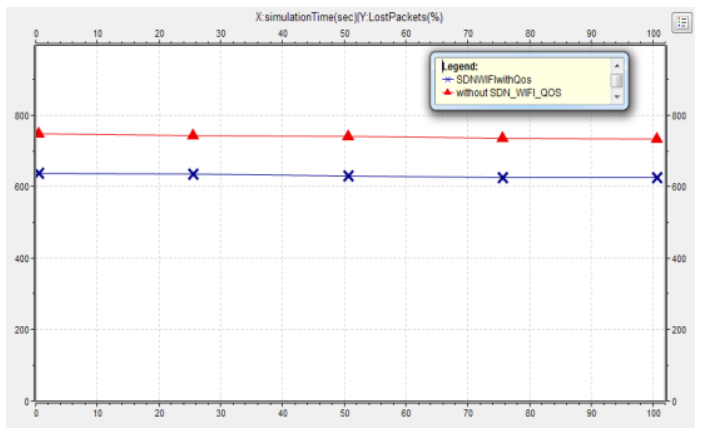

Fig. 7. Figlost Packets in Wi-Fi SDN-Free and SDN-Based Scenarios.

\section{E. MoS under Wi-Fi without and with SDN.}

Fig. 8 shows that MOS offered by the Wi-Fi approach without $\mathrm{SDN}$ is 2.2 while the approach based on Wi-Fi with SDN is about 3.2; it presents an indicator of increases in quality of voice transmission.

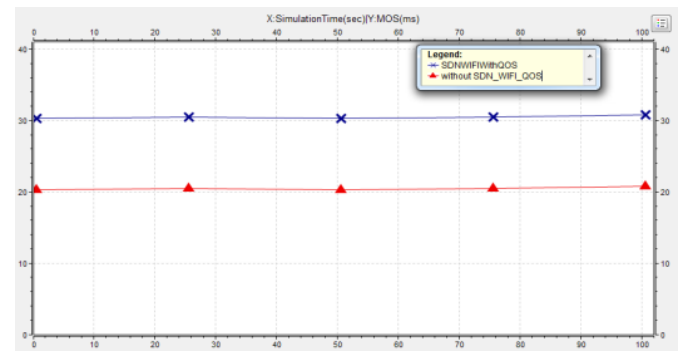

Fig. 8. Fig8. MOS in Wi-Fi SDN-free and SDN-based scenarios. 


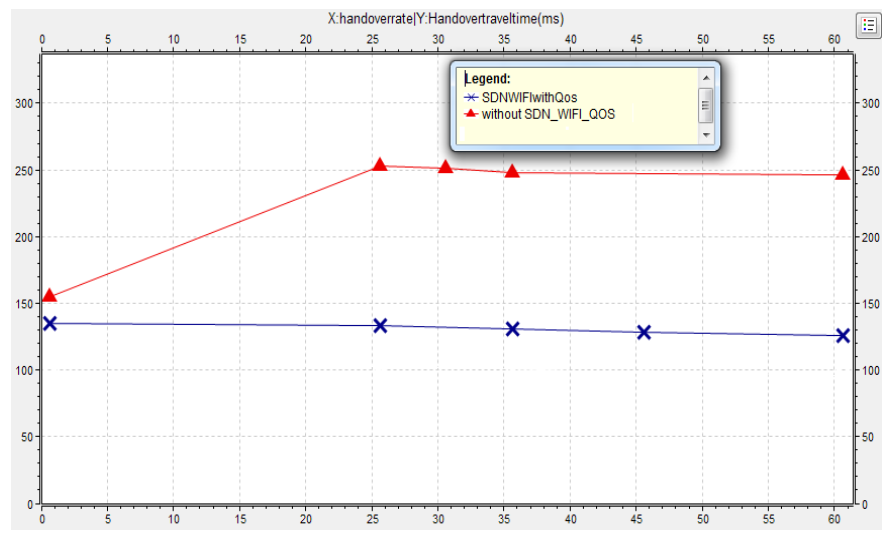

Fig. 9. Handover in Wi-Fi SDN-Free and SDN-Based Scenarios.

\section{HANDOVER UNDER WI-FI WITHOUT AND WITH SDN}

Fig. 9 presents two scenarios to describe node transfer signalling in a Wi-Fi network with SDN, it is stable during all the communication with a minimum of time $(13 \mathrm{~ms})$, it is inferior to Wi-Fi without SDN (25 ms), so SDN can benefit from the services of another cell instead of the old one. This allows the mobile station the ability to continue its ongoing communication with a minimum of interruption, knowing that the two cells involved are managed by one or more networks.

\section{CONCLUSION}

This article presents the improvement the Handover parameters and the QoS, under OMNeT 4.6++, that it is offered by the WiFi network by adding a new algorithm SDN, for $\mathrm{Wi}-\mathrm{Fi}$, which it is more preferable to $\mathrm{Wi}-\mathrm{Fi}$ without SDN.

This work is considered a part of another article that determines a contribution of $4 \mathrm{G}$ with $\mathrm{SDN}$ in terms of $\mathrm{HO}$ and QoS.

\section{REFERENCES}

[1] Fatima LAASSIRI, Mohamed MOUGHIT, Noureddine IDBOUFKER, "Improvement of Multiprotocol Label Switching Network's Performance using Software Defined Network Approach", IJSER, June -2018 .

[2] Jin-Shyan Lee, Yu-Wei Su, Chung-Chou Shen, “A Comparative Study of Wireless Protocols: Bluetooth, UWB, ZigBee, and Wi-Fi”, IEEE, 03 March 2008.

[3] Laizhong Cui, F. Richard Yu, and Qiao Yan, When Big Data Meets, "Software-Defined Networking: SDN for Big Data and Big Data for SDN", IEEE Network. January/February 2016.

[4] Henry Sinnreich, Alan B.Johnston, "Internet Communications Using SIP, Delivering VOIP and Multimedia Services with Session Initiation Protocol', Second Edition, books.google.com, 2012.

[5] A. Patel, K. Leung, M. Khalil, H. Akhtar, K. Chowdhury, RFC 4283, "Mobile Node Identifier Option for Mobile IPv6 (MIPv6)", November 2005.

[6] Jin-Shyan Lee, Yu-Wei Su, Chung-Chou Shen, "A Comparative Study of Wireless Protocols: Bluetooth, UWB, ZigBee, and Wi-Fi', IEEE,03 March 2008.
[7] Shailandra Kaushik, An overview of Technical aspect for WiFi Networks Technology, IJECSE, Volume1, Number 1.

[8] Anita Nikolich, "SDN Research Challenges and Opportunities", CODASPY '16: Proceedings of the Sixth ACM Conference on Data and Application Security and Privacy, March 2016.

[9] D. Tennenhouse and D. Wetherall, "Towards an active network architecture", ACM Computer Communication Review, 26(2), pp. 5-17, 1996.

[10] B. Schwartz, A. W. Jackson, W. T. Strayer, W. Zhou, R. D. Rockwell, and C. Partridge, "Smart Packets for active networks", IEEE Second Conference on Open Architectures and Network Programming Proceedings OPENARCH '99, IEEE xplore, pp. 90-97, 1999.

[11] Bruno Astuto A. Nunes, Marc Mendonca, Xuan-Nam Nguyen "A Survey of Software-Defined Networking: Past, Present, and Future of Programmable Networks", Volume: 16, Issue: 3, IEEE, February 2014.

[12] Alberto Bemporad, Antonio Bicchi, Giorgio Buttazzo, "Hybrid Systems: Computation and Control", 10th International Workshop, Proceedings, HSCC 2007, Pisa, Italy, April 3-5, 2007.

[13] A. Greenberg, G. Hjalmtysson, D.A. Maltz, A. Myers, J. Rexford, G. Xie, H. Yan, J. Zhan et H. Zhang, "A Clean Slate 4D Approach to Network Control and Management", ACM SIGCOMM Computer Communication Review, 35(5), pp. 41-54, 2005.

[14] M. Casado, M. J. Freedman, J. Pettit, J. Luo, N. McKeown et S. Shenker, "Ethane: Taking Control of the Enterprise", Proceedings of the Conference on Applications, Technologies, Architectures, and Protocols for Computer Communications, pp. 1-12, 2007.

[15] N. Gude, T. Koponen, J. Pettit, B. Pfaff, M. Casado, N. McKeown et S. Shenker, "NOX: Towards an Operating System for Networks", ACM SIGCOMM Computer Communication Review, 38(3), pp. 105-110, 2008.

[16] Fouad BENAMRANE, Etude des Performances des Architectures du Plan de Contrôle des Réseaux 'Software-Defined Networks, January 2017.

[17] Rohde-schwar, “White Paper 802.11ac Technology Introduction", page $6-2012$.

[18] https://fr.wikipedia.org/wiki/IEEE_802.11ac, January 2017.

[19] Doug Marshke, Jeff Doyle and Pete Moyer, "Software Defined Networking (SDN): Anatomy of OpenFlow Volume I", "SDN Essentials", June 2017.

[20] Marcial P Fernandez, "Comparing OpenFlow Controller Paradigms Scalability: Reactive and Proactive", IEEE 27th International Conference on Advanced Information Networking and Applications, 2013.

[21] Wendell Odom, SDN Termilogy from layered models, https://www.sdnskills.com/learn/ sdn-terms-01/, May 13, 2015.

[22] Evangelos Haleplidis, Stefano Salsano, "Overview of RFC7426: SDN Layers and Architecture Terminology", IEEE Softwarization, September 2017.

[23] Open Networking Foundation, "SDN Architecture Overview", Version 1.0 December 12, 2013.

[24] https://www.sdxcentral.com/sdn/definitions/inside-sdn-architecture/, January 2018.

[25] P. Bosshart, D. Daly, G. Gibb, M. Izzard, N. McKeown, J. Rexford, C. Schlesinger, D. Talayco, A. Vahdat, G. Varghese, "Walker, Public Review for Programming Protocol-Independent Packet Processors", ACM SIGCOMM Computer Communication Review, Volume 44, Number 3, July 2014.

[26] Jérôme Durand, "Le SDN pour les nuls", Cisco Systems, p8, JRES 2015. 